\title{
Conocimiento acerca de las voluntades anticipadas por parte del adulto mayor del Hogar San Pedro Claver 2015-2016
}

\section{Knowledge about the previous wills by the senior adult of the Home San Pedro Claver 2015-2016}

\author{
Stephanye Carrillo González, Jaime Lorduy Gómez
}

Programa

de

Instrumentación Quirúrgica, Corporación
Núñez. stephanye.carrillo@curnvirtual.edu.co

Universitaria

Rafael

\section{RESUMEN}

Introducción: las voluntades anticipadas "son instrumentos útiles para proyectar la protección del derecho a la autodeterminación de las personas, especialmente en situaciones en las cuales no son competentes para hacer juicios razonados". Este tema es relevante, ya que la escasa información y difusión de las voluntades anticipadas en los adultos mayores, acompañado de la ausencia de investigaciones y reglamentaciones nacionales permiten vulnerar los principios de la bioética entre los que sobresale el de autonomía. Actualmente en Cartagena no se conoce estudio alguno sobre las voluntades anticipadas en los distintos hogares geriátricos, por lo tanto se justifica este estudio. Con el objetivo de determinar el conocimiento en relación a las voluntades anticipadas del adulto mayor del Hogar San Pedro Claver de la Ciudad de Cartagena en los años 2015-2016. Métodos: el tipo de estudio es descriptivo, de corte trasversal. La obtención de datos se tuvo de acuerdo a la operacionalización de variables en una encuesta aplicada a 55 adultos mayores que se encontraban en el Hogar estudiado, según el criterio para escoger a los sujetos a investigar e información útil que permitió la formulación de conclusiones y recomendaciones. Para el análisis de la información se utilizó el programa estadístico SPSS versión 22. Para este estudio en cuanto a normas éticas en Investigación especialmente la Resolución 008430 de 1993 quien clasifica sin riesgos esta investigación. Resultados: los hallazgos de este estudio indican que los adultos mayores de este Hogar prevalece el sexo masculino, tienen procedencia del área urbana, son en la mayoría solteros, tienen un nivel de escolaridad entre primaria incompleta y secundaria incompleta, otro aspecto es que casi la mitad no recibe ayuda económica, esto se relaciona con las voluntades anticipadas ya que respondieron negativamente a la hora de autorizar a sus familiares las decisiones frente al destino final de su cuerpo y sus órganos, trasfusiones sanguíneas, cuidados paliativos y testamento vital. Conclusiones: existe desconocimiento en el tema de voluntades anticipadas por parte de los adultos mayores y se resalta que ellos piensan que su familia no debe tomar decisiones por ellos. En otro aspecto se hace necesario la educación al personal responsable que interviene y acompaña a los pacientes geriátricos, para que este reciba todo la instrucción y alternativas que ellos poseen como sujetos autónomos y que haya el necesario acompañamiento en la toma de decisiones. 
Palabras Clave: directivas anticipadas, geriatría, voluntad en vida.

\section{ABSTRACT}

Introduction: Introduction: the anticipated wills "are useful instruments to project the protection of the right to self-determination of people, especially in situations in which they are not competent to make reasoned judgments". This issue is relevant, since the scarce information and dissemination of the wills anticipated in the elderly, accompanied by the absence of national research and regulations allow to violate the principles of bioethics, among which autonomy stands out. Currently in Cartagena there is no known study on the anticipated wills in the different nursing homes, therefore this study is justified. With the objective of determining the knowledge in relation to the anticipated wills of the older adult of Hogar San Pedro Claver of the City of Cartagena during 2015-2016. Methods: a cross-sectional descriptive study was carried out. The data collection was based on the operationalization of variables in a survey applied to 55 older adults who were in the Home studied, according to the criteria for choosing the subjects to investigate and useful information that allowed the formulation of conclusions and recommendations. For the analysis of the information, the statistical program SPSS version 22 was used. For this study regarding ethical standards in Research, especially Resolution 008430 of 1993, which classifies this investigation without risk. Results: the findings of this study indicate that older adults of this household prevail male sex, are from the urban area, are mostly single, have a level of education between incomplete primary and incomplete secondary, another aspect is that almost half does not receive financial aid, this is related to the anticipated wills since they responded negatively when authorizing their families decisions regarding the final destination of their body and organs, blood transfusions, palliative care and living will. Conclusions: there is ignorance on the subject of anticipated wills on the part of the elderly and it is emphasized that they think that their family should not make decisions for them. In another aspect it is necessary to educate the responsible personnel who intervene and accompany the geriatric patients, so that they receive all the instruction and alternatives that they have as autonomous subjects and that there is the necessary accompaniment in the decision making process.

Keywords: advance directives, geriatrics, living wills.

\section{INTRODUCCIÓN}

Las voluntades anticipadas "son instrumentos útiles para proyectar la protección del derecho a la autodeterminación de las personas, especialmente en situaciones en las cuales no son competentes para hacer juicios razonados. Se trata de un documento en el que una persona plasma su decisión, adoptada de manera libre, ilustrada y autónoma, frente a una situación de salud en la que no sean predicables su aptitud ni su capacidad para discernir sobre conductas médicas, o en incapacidad física, de abstención terapéutica o de garantía de no ser expuesta a sufrimientos innecesarios y fútiles o de ponerle fin a su propia existencia" (1). 
Hasta ahora se han utilizado el de testamento vital, que la mayoría de los autores rechaza, pues no es un documento ni de cumplimiento obligatorio ni que se tenga en cuenta después de la muerte. Otro término muy usado en Estados Unidos es el de directrices previas o anticipadas, a tono con la importancia que le dan a la autonomía del paciente que decide previamente lo que quiere se le realice o no en la atención de salud. El tercer nombre, más usado actualmente en España y Latinoamérica, es el de voluntades anticipadas, y quizás es el más apropiado a nuestro ámbito (2).

Según los autores Bolívar Góez y Gómez Córdoba mencionan que "la relación de los profesionales de la salud con los pacientes tiene como piedra angular el respeto del derecho de las personas a auto determinarse, a tomar decisiones libres e informadas respecto a su vida, salud y cuerpo. El final de la vida es uno de los más importantes momentos vitales en el cual es definitivo garantizar que el paciente pueda tomar una serie de decisiones sobre múltiples aspectos, como son el deseo o no de ser informado, aceptar o no medidas terapéuticas y de cuidado paliativo, rechazar aquellas que son fútiles, definir quién puede acceder a sus datos clínicos o quién representara su voluntad; esto con el fin de proteger su derecho a una muerte digna. No obstante, en esta parte de la vida es frecuente que el paciente se encuentre aquejado por patologías que alteran su competencia para decidir, lo cual nos obliga a recurrir a instrumentos que protejan y proyecten su autonomía en el tiempo, como es el caso de las voluntades anticipadas" (1).

"La progresión de una enfermedad crónica, el diagnóstico de una enfermedad incurable, la constatación del empeoramiento de la salud, son algunas de las múltiples situaciones que exponen a la persona y a sus familiares y allegados a una situación amenazante y difícil. Desde distintos ámbitos del conocimiento y también por parte de la sociedad se reclama, cada vez con más intensidad, la necesidad de atender profesional y humanamente a las personas en la etapa final de su vida" (3).

"Los documentos de voluntades anticipadas (DVA), que han tomado cierta relevancia en las últimas décadas, constituyen una forma más de empoderamiento del paciente que toma un rol protagónico en el proceso de su propia enfermedad. No obstante, es pertinente señalar que, a pesar de los esfuerzos por difundir su utilidad, en la práctica no han tenido la acogida esperada" (4). Interesante mirar esta problemática ya que la escasa información y difusión de las voluntades anticipadas, acompañado de la ausencia de investigaciones y reglamentaciones nacionales permiten vulnerar los principios de la bioética entre los que sobresale el de autonomía, pues los adultos mayores en ocasiones se les trasgreden sus derechos, quizás por desconocimiento o tal vez por apatía.

"Son diversos los contenidos u objetos de la voluntad anticipada: el cuidado paliativo, la aceptación o el rechazo de tratamientos futuros, la readecuación o la limitación del esfuerzo terapéutico, la designación de una persona que represente al paciente, cuando a este no le sea posible expresar su voluntad, para la donación de órganos (con fines 
médicos, de docencia o de investigación); asimismo, el acceso de información a terceros, el lugar de la muerte, la aceptación o el rechazo de apoyo religioso y la terminación anticipada de la vida. El objeto de las voluntades estará limitado por el marco jurídico vigente en cada país" (1). En Colombia se contextualizan las voluntades anticipadas en antecedentes jurídicos en los derechos humanos, la Constitución Política de Colombia y reglamentaciones como: Ley 1733 de 2014 mediante la cual se regulan los cuidados paliativos (5). Sin embargo la problemática es "la escasa difusión en la población general del derecho a suscribir voluntades anticipadas, la escasa formación de los profesionales de la salud sobre el tema y la ausencia de registros nacionales respaldados por el Estado que faciliten el acceso de los profesionales de la salud a sistemas de información en los cuales reposen la totalidad de las voluntades anticipadas" (1).

Actualmente en Cartagena no se conoce estudio alguno en los distintos hogares geriátricos sobre las voluntades anticipadas, por lo tanto se justifica la investigación.

\section{MÉTODOS}

El tipo de estudio es descriptivo, de corte trasversal y en relación con el tiempo prospectivo. La obtención de datos se tuvo de acuerdo a la operacionalización de variables en una encuesta aplicada a 55 adultos mayores en el hogar, que se encontraban competentes para responder, y aplicaron al estudio según el criterio para escoger a los sujetos a investigar.

Teniendo en cuenta los datos encontrados en el instrumento aplicado se creó una base de datos en el programa Excel, los cuales se categorizarán según la naturaleza de las variables: en cualitativas y cuantitativas, según el grado de medición: en nominales y ordinales, para una mejor tabulación y comprensión de los datos. Los cálculos se dieron de acuerdo a las medidas de frecuencia, los cuales se graficaron en gráficas de pasteles y tabla. Para el análisis de la información se utilizó el programa estadístico SPSS versión 22.

Para este estudio en cuanto a normas éticas en Investigación se tuvo en cuenta a nivel internacional en materia de investigación biomédica se fundamentó en los criterios del Código de Núremberg (6), Declaración de Helsinki (7) y la reglamentación colombiana vigente entre ella la Resolución 008430 de 1993 (8). Por la misma naturaleza de la investigación y la manera de cómo se recogieron los datos se determinó una investigación sin riesgos, ya que se contó con el aval de la Institución y el consentimiento de los adultos mayores a encuestar, respetando la confidencialidad de los mismos.

\section{RESULTADOS}


En cuanto a las características demográficas de la población de estudio es interesante anotar que los adultos mayores prevalecen el sexo masculino, tienen procedencia del área urbana, son en la mayoría solteros, tienen un nivel de escolaridad entre primaria incompleta y secundaria incompleta, otro aspecto es que casi la mitad no recibe ayuda económica (ver tabla 1).

\begin{tabular}{|c|c|c|}
\hline Variables de Identidad & Categoría & Porcentaje \\
\hline \multirow{2}{*}{ Sexo } & Masculino & $55 \%$ \\
\hline & Femenino & $45 \%$ \\
\hline \multirow{2}{*}{ Procedencia } & Urbana & $80 \%$ \\
\hline & Rural & $20 \%$ \\
\hline \multirow[t]{5}{*}{ Estado civil } & Soltero & $69 \%$ \\
\hline & Casado & $13 \%$ \\
\hline & Unión libre & $5 \%$ \\
\hline & Divorciado(a) & $2 \%$ \\
\hline & Viudo(a) & $11 \%$ \\
\hline \multirow{5}{*}{ Sustento económico } & Pensionado & $5 \%$ \\
\hline & Renta propia & $7 \%$ \\
\hline & Ayuda familiar & $24 \%$ \\
\hline & No tiene ayuda económica & $49 \%$ \\
\hline & Otra & $15 \%$ \\
\hline \multirow{7}{*}{ Nivel educativo } & Primaria completa & $24 \%$ \\
\hline & Primaria incompleta & $27 \%$ \\
\hline & Secundaria completa & $20 \%$ \\
\hline & Secundaria incompleta & $15 \%$ \\
\hline & Estudios técnicos & $5 \%$ \\
\hline & Universitarios & $4 \%$ \\
\hline & No ha estudiado & $15 \%$ \\
\hline
\end{tabular}

Tabla 1. Características de identidad de los adultos mayores del Hogar San Pedro Claver

Se resalta que un $46 \%$ los adultos mayores pensaría antes de firmar un documento anticipado en el que se decidiría libremente el destino sus órganos una vez fallecido (ver figura 1). En la figura 2 se observa que un $49 \%$ de la población encuestada si autorizaría a su familia en el momento de decisiones en el momento donde su vida esté en riesgo para el manejo de medicamentos. 

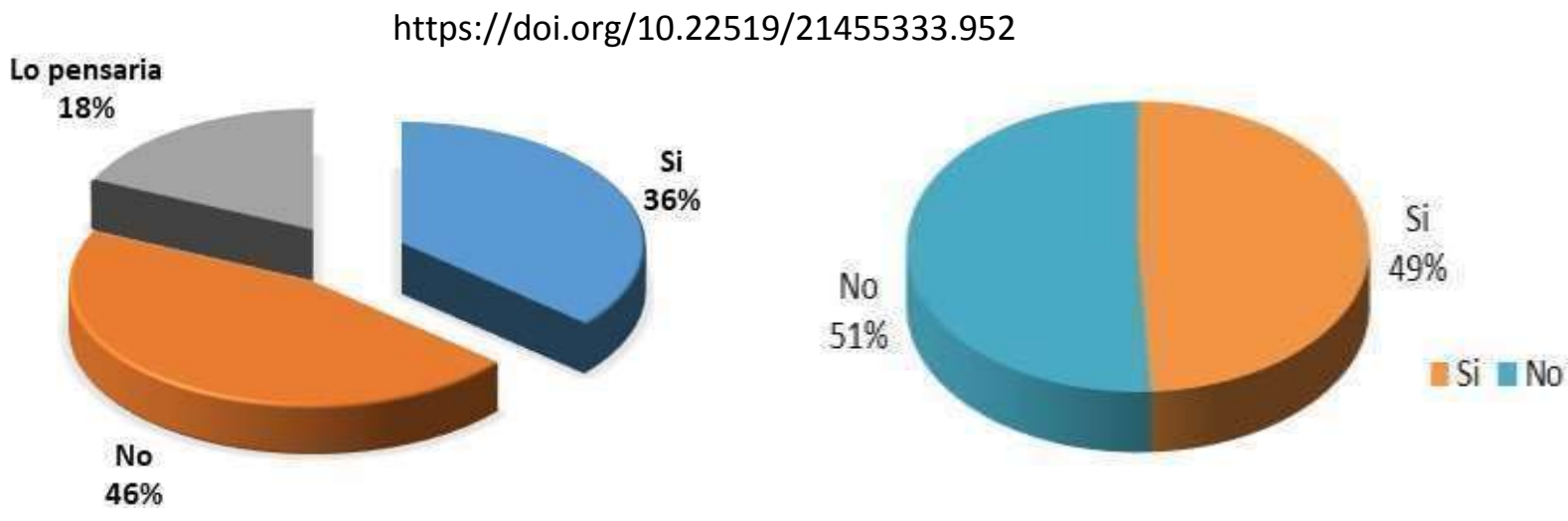

Figura 1. Porcentaje de adultos mayores del

Figura 2. Porcentaje de adultos mayores del Hogar San Pedro Claver que firmaría un Hogar San Pedro Claver que autorizaría a su documento en el que decidiría libremente el familia a tomar decisiones para el manejo de destino final de su

cuerpo y órganos una vez medicamentos en un momento donde esté fallecido

en riesgo su vida

Adicionalmente, el $27 \%$ de la población encuestada si autorizaría a su familia para que tomen decisiones en un momento en que su vida esté en riesgo para la donación de órganos (ver figura 3). El $44 \%$ si autorizaría a su familia para que tomen decisiones en el momento en que esté en riesgo su vida para transfusiones sanguíneas (ver figura 4).
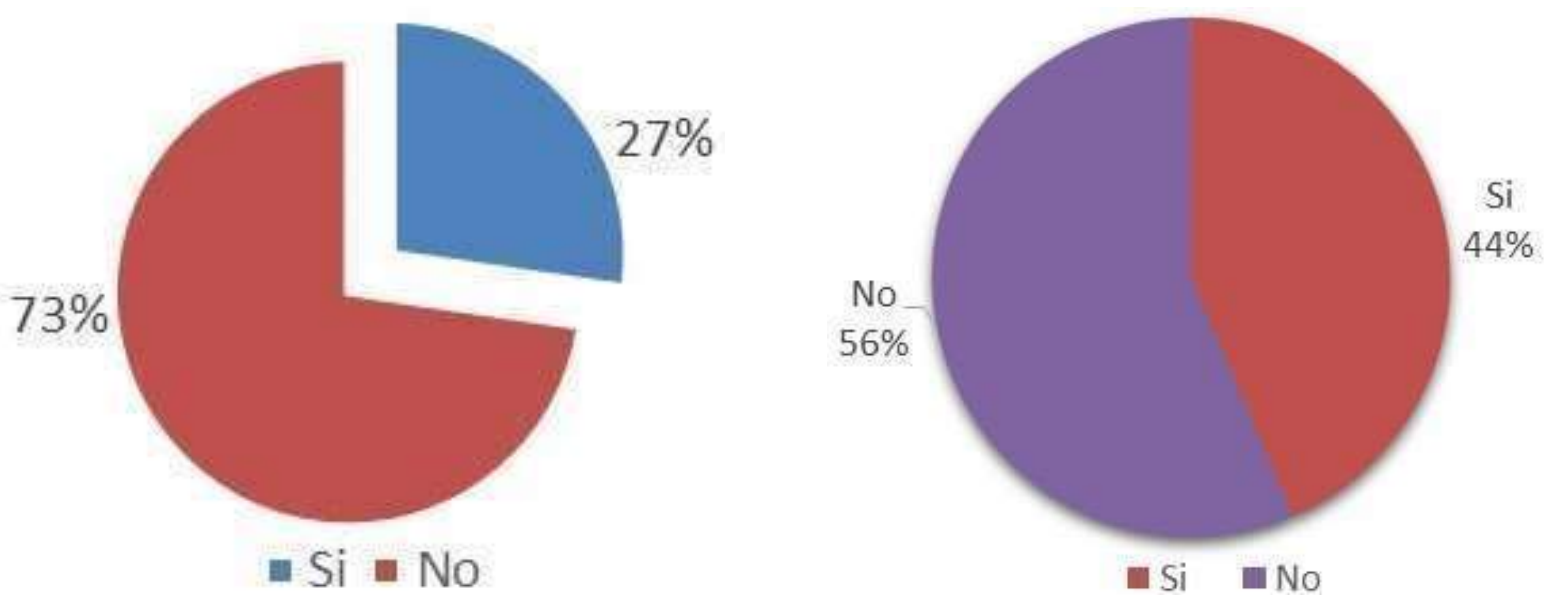

Figura 3. Porcentaje de adultos mayores del

Hogar San Pedro Claver que autorizaría a su familia en

la toma de decisiones para la donación de órganos en un momento donde esté en riesgo su vida

Figura 4. Porcentaje de adulto mayor del Hogar San Pedro Claver que autorizaría a su familia en la toma de decisiones para las 
En la figura 5 se nota que el $38 \%$ de la población encuestada si autorizaría a su familia para la práctica de reanimación cardiopulmonar en el momento en que esté en riesgo su vida. Por otra parte, el $42 \%$ de la población encuestada si autorizaría a su familia para la utilización de cuidados paliativos en el momento en que su vida esté en riesgo (figura $6)$.

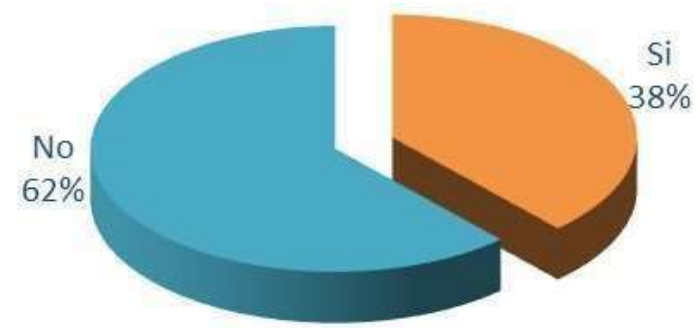

Si $=$ No

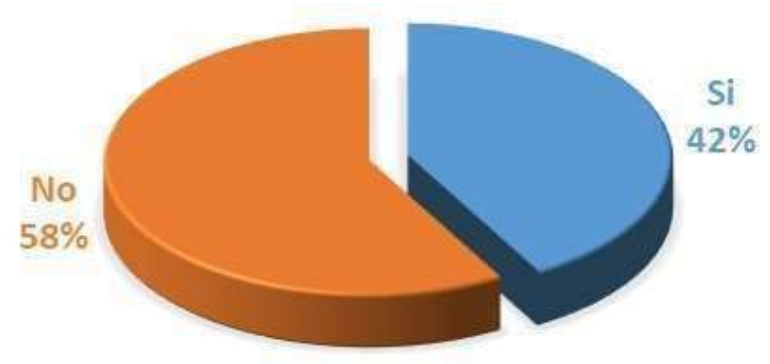


Figura 5 Porcentaje de adulto mayor del Hogar San Pedro Claver que autorizaría a su familia en la toma de decisiones para las fami reanimaciones cardiopulmonar en un momento en un momento donde esté en riesgo su vida donde esté en riesgo su vida
Figura 6 Porcentaje de adulto mayor del Hogar San Pedro Claver que autorizaría a su la utilización de Cuidados Paliativos

En la figura 7 se observa que un $44 \%$ de la población encuestada si autorizaría un testamento vital en un momento donde esté en riesgo su vida; mientras que el $60 \%$ de la población encuestada está de acuerdo con la reanimación artificial (figura 8).

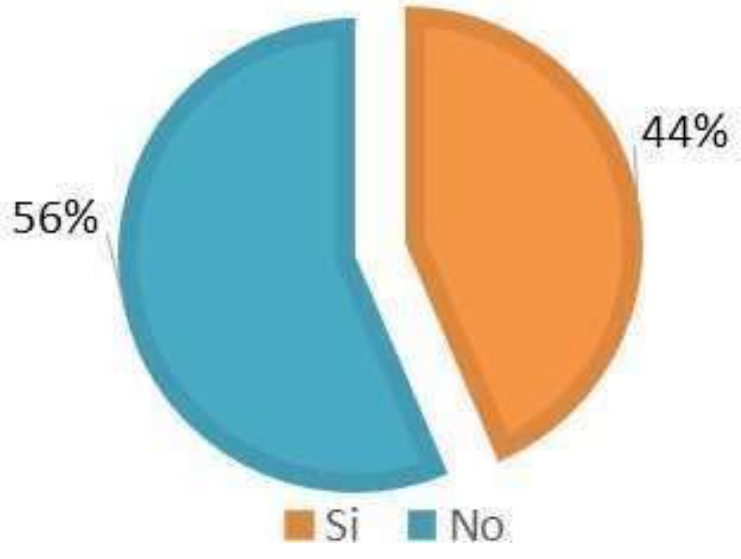

Figura 7. Porcentaje de adulto mayor del

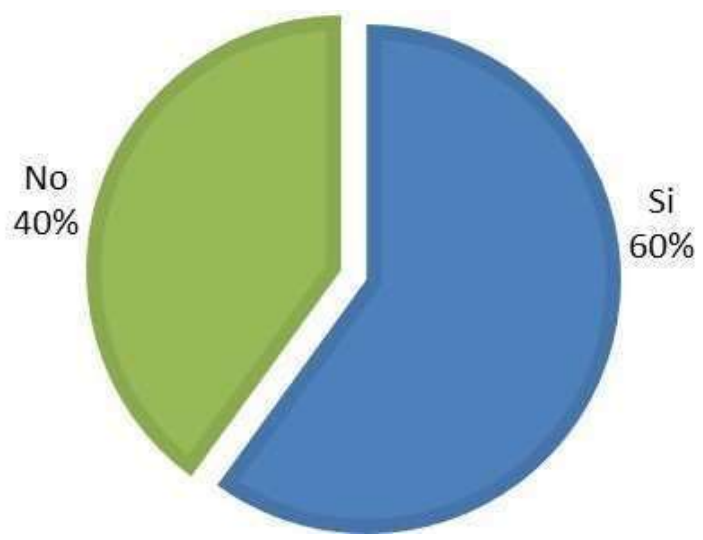

Figura 8. Porcentaje de adulto mayor del Hogar San Pedro Claver que autorizaría un Hogar San Pedro Claver que está de acuerdo testamento vital con la reanimación artificial en una situación

clínica difícil

\section{DISCUSIÓN}

Los resultados de esta investigación son comparables con los reportados en la literatura. Se encontró en el presente estudio un desconocimiento de las voluntades anticipadas por parte de los adultos mayores, esto se asemeja a una investigación realizada por Cristina Nebot et al en España donde "la mayoría de los otorgantes de voluntades anticipadas profesan una determinada confesión y rechazan que se les practiquen determinados tratamientos. La mayoría de los profesionales no comprueba si los enfermos en situación crítica han otorgado voluntades anticipadas. La diversidad de criterios entre comunidades complica el ejercicio del derecho a expresar estas voluntades" (9). Ante este tema agrega Cristina Quijada y María Tomas y Garrido (10) "La idea de un documento para que el paciente rechace, de manera generalizada e hipotética, tratamientos concretos cuyo alcance y consecuencias desconoce, ha 
demostrado no tener la aceptación social esperada ni en la población ni en un sector muy importante de los profesionales sanitarios".

Un factor en esta investigación que se relaciona con el desconocimiento acerca del tema de las voluntades anticipadas, es la ausencia de los familiares, se evidencia cuando al responder un gran porcentaje de los adultos mayores no autorizaría a su familia la decisión de reanimación cardiopulmonar ante un momento determinado, pero más adelante en el cuestionamiento se le indaga si está de acuerdo con la reanimación artificial y un mismo porcentaje contesto afirmativamente; se asume la dualidad de las respuestas y el desconcierto del tema, al hecho de estar lejos de su familia, como diría León Correa: "La confusión entre algunas situaciones clínicas -rechazo de tratamientos, limitación de tratamientos, peticiones de eutanasia- lleva a algunos al rechazo sin más de las voluntades anticipadas, o impulsa a otros a defenderlas como "paso previo" a una legalización de la eutanasia" (2). La solución ante esto, lo plantea Bolívar, et al, (1) y su argumentación es que "en esta parte de la vida es frecuente que el paciente se encuentre aquejado por patologías que alteran su competencia para decidir, lo cual nos obliga a recurrir a instrumentos que protejan y proyecten su autonomía en el tiempo, como es el caso de las voluntades anticipadas". Deduciendo con esto, que estos recursos incomprensibles podrían ser su ayuda en un momento determinado. Con razón decía Josep Arimany-Manso y colaboradores (11) "El entorno de los pacientes suele desconocer el documento de Voluntades Anticipadas, sin embargo, suele existir un gran interés por el mismo. Llegado el momento, la inexistencia del DVA genera mucha inquietud al entorno afectivo del paciente, que se ve con la responsabilidad de tomar decisiones por el paciente y no siempre existe consenso entre los familiares respecto a lo que se debe hacer, lo cual genera angustia, desazón e incluso culpabilidad".

En otro aspecto en este estudio más de la mitad de los adultos mayores opinaron que no utilizarían los Cuidados paliativos ante una enfermedad terminal, y esto da a entender el desconocimiento del tema, ya que este tipo de cuidados están inmersos en las voluntades anticipadas y que abren paso a una era diferente, donde se toma conciencia que la medicina tradicional y clínica no lo es todo, más bien es un reconocimiento de los límites de esta. Ante esto la Organización Mundial de la Salud dice que "Los cuidados paliativos constituyen un planteamiento que mejora la calidad de vida de los pacientes (adultos y niños) y sus allegados cuando afrontan problemas inherentes a una enfermedad potencialmente mortal. Previenen y alivian el sufrimiento a través de la identificación temprana, la evaluación y el tratamiento correctos del dolor y otros problemas, sean estos de orden físico, psicosocial o espiritual". De igual forma la OMS en su apreciación aporta que "Afrontar el sufrimiento supone ocuparse de problemas que no se limitan a los síntomas físicos. Los programas de asistencia paliativa utilizan el trabajo en equipo para brindar apoyo a los pacientes y a quienes les proporcionan cuidados. Esa labor comprende la atención de necesidades prácticas y apoyo psicológico a la hora del duelo. 
La asistencia paliativa ofrece un sistema de apoyo para ayudar a los pacientes a vivir tan activamente como sea posible hasta la muerte" (12).

De todo lo anterior es importante capacitar a los cuidadores de estos adultos de tal forma que "las voluntades anticipadas se enmarcan en la planificación de cuidados, entendida como un proceso positivo de reflexión e implicación de los ciudadanos y ciudadanas en las decisiones relativas a su salud" (3), y aún más abrir los espacios para el dialogo de estos temas. Muy bien decía ante lo anterior, Laura Florencia Billi (13) "Los procesos de comunicación y la deliberación entre pacientes, profesionales y seres queridos constituyen unos de los aspectos más importantes del cuidado y permiten que el fin de la vida sea comprendido como un proceso natural y como una experiencia llena de sentido. El fracaso de las propuestas clásicas de los documentos de directivas anticipadas se ha debido en gran medida al hecho de que no se ha otorgado la importancia que merece a la comunicación entre los pacientes y el resto de las personas involucradas en la toma de decisiones sobre su salud".

Así mismo Romero Rodríguez y colaboradores (3) opinan que "Los objetivos del equipo multidisciplinar que trabaja con el enfermo y su familia deben converger en facilitar la adaptación de ambos a esta difícil situación, promoviendo el máximo bienestar posible y amortiguando el sufrimiento".

Para poner en práctica esta vertiente de aliviar, es necesario coordinar los esfuerzos de muchos profesionales sanitarios, enfrentando una atención y cuidado y esa sí que es una tarea dura, especialmente porque se necesitan personas con una específica y elevada calidad humana y científica, ante eso no se puede improvisar. "Los profesionales de la salud deben conocer la legislación vigente en materia del principio de autonomía del paciente, teniendo la responsabilidad profesional legal y ética de asegurar la protección del paciente en caso de vulnerabilidad y el máximo respeto a su autonomía, por lo que es necesaria una tarea divulgativa y pedagógica ante los profesionales de la salud, las administraciones y la población general" (11).

\section{CONCLUSIONES}

Los hallazgos de este estudio indican claramente que hay un desconocimiento de las voluntades anticipadas por parte de los adultos mayores de este hogar en Cartagena. Si bien es cierto la autonomía de estos adultos mayores se ve disminuida por el abandono, desesperanza, tristeza y hasta depresión que poseen.

La mitad de los adultos mayores no recibe ayuda de su familia, esto se relaciona con el bloqueo ante temas como testamento vital y a su vez a las voluntades anticipadas, ya que respondieron negativamente a la hora de autorizar a sus familiares las decisiones 
frente al destino final de su cuerpo y sus órganos, trasfusiones sanguíneas, cuidados paliativos, reanimación cardiopulmonar y testamento vital.

Es importante el hecho de plantear acompañamiento ante el sufrimiento y el dolor, rescatando en ellos el apercibimiento de la vida más allá de su cuerpo (el valor espiritual de ellos), la claridad ante esto es sumamente sustancial para estos adultos mayores, sobre todo con mayor énfasis en la cobertura emocional, en este caso por parte de los cuidadores que puedan comprenderlos como persona, no juzgándolos, no discriminándolos, sino planteándoles soluciones que le demanden en cierta forma una mirada en esos últimos días como una oportunidad para subsanar temas personales y familiares.

Puesta en escena toda esta situación se hace necesario la educación ante el tema de Voluntades anticipadas; sin embargo hay que actualizarse en los parámetros que se establezcan en el país, para que el personal responsable que interviene y acompaña a los pacientes geriátricos reciba toda la instrucción y alternativas que los adultos mayores poseen como sujetos autónomos, y que frente al tema haya el necesario acompañamiento, abriéndose al dialogo concienzudo, siempre apuntando a que estos sean dignificados como persona y reciban toda la atención humanizada que ameritan.

Agradecimientos a los docentes y estudiantes de los programas de Instrumentación Quirúrgica y Medicina de la Corporación Universitaria Rafael Núñez especialmente a las profesoras Ruby Muñoz Baldiris y Dilia Aparicio Marenco que coordinan Investigación en cada programa respectivamente. A los estudiantes de medicina Luis David Acevedo Cataño, Diana Marcela Acosta Agamez, Mauricio Alfredo Caez Pérez, Kelly Andrea Contreras Licero, Jennifer Díaz Blanco y a los estudiantes de Instrumentación quirúrgica Aylin Teresa Morillo Herrera, Karla Liliana Arteaga Marín quienes aportaron en la elaboración del proyecto, al igual que las directivas del Hogar San Pedro Claver por autorizar la intervención.

\section{REFERENCIAS}

1. Bolívar Góez, Gómez Córdoba A. Voluntades anticipadas al final de la vida. Una aproximación desde la regulación colombiana y en el derecho comparado. Revista Latinoamericana de Bioética. 2016 Junio; 16(1).

2. León Correa. Las voluntades anticipadas:análisis desde las experiencias en ética clínica. Revista colombiana de Bioética. 2008 Diciembre; 3(2).

3. Romero Rodríguez, López Tapia , De Rosales Martínez M, Martin Sánchez MÁ, Romero Cotelo , Galvez Mateos. El manejo de la información en Cuidados Paliativos. Psicooncología. 2013; 10(2-3).

4. Malespina. Cuestiones jurídicas al final de la vida. Persona y Bioética. 2017 Julio-Diciembre; 21(2).

5. Presidencia de la República. Ley 1733 de 2014 Regulación de Cuidados paliativos en Colombia. ; 8 septiembre de 2014.

6. Mainetti JA. The Nuremberg Code. Traducción adaptada en 1989. Quirón.La Plata Argentina: Ética médica; 1947. 
7. World Medical Association. Declaration of Helsinki. 64 ${ }^{\mathrm{a}}$ Asamblea General. Fortaleza Brasil:; Octubre 2013.

8. Ministerio de Salud. Resolución N008430 de 1993 Normas Cientificas, tecnicas y administrativas para la investigación en salud. Santafé de Bogotá: Ministerio de Salud; 1993.

9. Nebot, Ortega, Mira, Ortiz. Morir con dignidad. Estudio sobre voluntades anticipadas. Gaceta sanitaria. $2010 \mathrm{Nov} / \mathrm{dic} ; 24(6)$.

10. Quijada-González C, Tomás y Garrido G. Testamento vital: conocer y comprender su sentido y significado. Persona y Bioética. 2014 Julio-Diciembre; 18(2).

11. Arimany-Manso, Aragonès-Rodríguez, Gómez-Durán EL, Galcerán, Martin-Fumadó, TorralbaRosselló. El testamento vital o documento de voluntades anticipadas. Consideraciones médico-legales y análisis de la situación de implantación en España. Revista Española de Medicina Legal. 2017 EneroMarzo; $43(1)$.

12. Organización Mundial de la Salud. OMS. Cuidados paliativos. [Online].; 2015 [cited 2016 Mayo 02. Available from: www.who.int/mediacentre/factsheets/fs402/es/.

13. Florencia Belli L. El debate en torno a la validez de las directivas anticipadas en salud. Persona y bioética. 2014 Julio-Diciembre; 18(2). 